\title{
Afro-christianisme et politique de l'identité : l'église du christianisme Céleste Versus celestial church of christ
}

André Mary

\section{(2) OpenEdition \\ Journals}

Édition électronique

URL : http://journals.openedition.org/assr/214

DOI : $10.4000 /$ assr.214

ISSN : $1777-5825$

Éditeur

Éditions de l'EHESS

\section{Édition imprimée}

Date de publication : 1 avril 2002

Pagination : 45-56

ISBN : 2-222-96718-X

ISSN : 0335-5985

Référence électronique

André Mary, «Afro-christianisme et politique de l'identité : l'église du christianisme Céleste Versus celestial church of christ », Archives de sciences sociales des religions [En ligne], 118 | avril - juin 2002, mis en ligne le 03 juin 2005, consulté le 19 avril 2019. URL : http://journals.openedition.org/assr/214 ; DOI : $10.4000 /$ assr.214 


\title{
AFRO-CHRISTIANISME ET POLITIQUE DE L'IDENTITÉ : L'ÉGLISE DU CHRISTIANISME CÉLESTE VERSUS CELESTIAL CHURCH OF CHRIST
}

\author{
À propos de : \\ SURGY (Albert de), L'Église du Christianisme \\ Céleste. Un exemple d'Église prophétique au Bénin, \\ Paris, Karthala, 2001, 332 p. \\ ADOGAME (Afeosemime U.), Celestial Church of \\ Christ, The Politics of Cultural Identity in a West \\ African Prophetic - Charismatic Movement, Peter \\ Lang, Studies in the Intercultural History of \\ Christianity, $\mathrm{n}^{\circ} 115,1999,251 \mathrm{p}$.
}

Comparée à l'histoire ivoirienne des Églises issues de la grande épopée prophétique d'Harris, à la promotion nationale des Églises kimbanguistes au Zaïre, ou encore à la dimension transrégionale des Églises zionistes d'Afrique du Sud, l'expansion internationale, et d'abord interafricaine, de l'Église prophétique du Christianisme Céleste reste un phénomène étonnant.

L'ouvrage d'Albert de Surgy est la première étude d'ensemble publiée en français sur cette Église chrétienne africaine d'origine béninoise bien connue des spécialistes anglophones des AIC (African Independent Churches) du fait de son expansion considérable au Nigeria, et de sa forte présence dans les diasporas africaines d'Europe et des États-Unis. L'entrée « béninoise » que nous propose l'auteur rend hommage, à juste titre, aux origines de cette Église fondée au Dahomey en 1947 par le Prophète Oschoffa, et au rôle historique de la "paroisse-mère » de Porto-Novo. Une telle entrée ne doit pas faire oublier cependant que, selon ses propres sources, dans les années 1990, le Nigeria (où l’Église est implantée depuis 1952 et où le prophète fondateur s'installe définitivement en 1977) comptait déjà cinq fois plus de fidèles que le Bénin, et que la Côte d'Ivoire elle-même (où l'Église apparaît dès 1950) contient sans doute aujourd'hui plus de fidèles (ivoiriens, et pas seulement des migrants nigérians ou béninois) qu'au Bénin. Avec ses deux mille paroisses, et ses ramifications mondiales, nul doute que le Diocèse du Nigeria - n'en déplaise à la « paroisse-mère » et à la «petite nation béninoise » - a une position dominante au sein de cette Église. De ce point de vue l'étude publiée 
par A. U. Adogame en 1999 - un travail universitaire de grande qualité soutenu à l'Université de Bayrouth et nourri d'un triple terrain (Nigeria, Angleterre et Allemagne) - se révèle très complémentaire. Cette étude apporte un éclairage décisif sur l'histoire et les développements récents de l'Église au Nigeria, où elle s'est imposée comme l'Église Aladura (Église des «priants », appellation propre aux Églises indépendantes du pays Yoruba) la plus importante et la plus attractive du pays, et sur les formes de son internationalisation (sites Web et autres). Rappelons que Rosalind Hackett avait déjà consacré dès 1978 sa thèse aux transformations historiques et sociologiques du charisme prophétique au sein de cette Église ${ }^{1}$.

Parler d'Église « prophétique », comme le fait A. de Surgy, se conçoit en partie $\mathrm{du}$ fait que son fondateur, d'éducation méthodiste, se disait Prophète (mais aussi Pasteur, ce qui signifiait pour lui chef de l'Église), mais aussi parce qu'une de ses ressources principales dans sa lutte contre les forces du mal est l'activité « prophétique » exercée par des agents attitrés, voyants, visionnaires, ou songeurs. Ce charisme d'institution ne justifie pas selon l'auteur la moindre confusion avec les Églises charismatiques, et encore moins avec la mouvance protestante, évangélique ou pentecôtiste (qualifiée de « rigoriste »), qui stigmatise les Célestes comme d'affreux païens ou féticheurs compromis avec les pratiques du vodou. Le fait est incontestable, surtout dans le contexte béninois, mais c'est en partie le souci de rompre avec cette image qui conduit justement certains leaders réformistes, au Nigeria ou en Côte d'Ivoire, et même au Bénin, à affirmer le caractère «pentecôtiste » ou « charismatique » de leur Église en insistant sur la puissance et l'efficacité de l'Esprit Saint qui sont au fondement de toutes leurs pratiques et sur les ressources qu'offrent les dons de prophétie, de guérison, et de miracle pour tous les Célestes. Les réserves formulées par rapport à la liturgie "bruyante » et « spectaculaire » de certains évangéliques, ou l'absence d'intérêt pour le «bla-bla glossolalique » (sic) auquel les Célestes préfèrent, il est vrai, d'autres manifestations plus corporelles des effluves de l'Esprit comme les transes visionnaires, les tremblements des corps agités, ou les prières «cacophoniques", sont-ils à ce point des traits discriminants qui interdisent de penser « une contamination par les pratiques pentecôtistes $»^{2}$ ? L'évolution de l'Église nigériane que nous retrace A. U. Adogame témoigne au contraire d'un mouvement manifeste de "pentecôtisation » qui semble aller de pair avec la mondialisation de l'Église.

Le rattachement de l'ECC (CCC, pour les anglophones, Celestial Church of Christ) aux Églises dites aladura d'origine Yoruba (al adua, ceux qui prient) ne semble pas non plus d'une grande utilité pour A. de Surgy, ce qui ne manque pas de surprendre, d'abord sur le fond, au regard de l'importance accordée à l'efficacité de la prière dans sa propre étude (deux chapitres au moins recensent, sur la base

${ }^{1}$ Hackett R.I.J., Explanation, Prediction and Control - The raison d'être of a West African Independant Church - The Celestial Church of Christ, M.Phil. Thesis, King's College, Université de Londres, 1978, une thèse au titre très hortonien mais à la problématique très wébérienne résumée dans "Thirty Years of Growth and Change in a West African Independent Church: A Sociological Perspective », in Hackett, ed, New Religious Movements in Nigeria, New-York, Edwin Mellen, 1987, pp. 161-177.

${ }^{2}$ Alfred DE SuRGY, [2001] note 1, p. 143. Dans le numéro « spécial Cinquantenaire » (septembre 1997) du journal La Dernière Barque, auquel notre auteur se réfère régulièrement, un article faisant le point sur «Ce qu'est l'ECC et ce qu'elle n'est pas » commence par ces mots : «L'Église du Christianisme Céleste est une Église chrétienne primitive de type pentecôtiste ... » (p. 6) La contamination est bel et bien engagée dans les têtes autant que dans les corps. 
d'un corpus significatif, les formes et types de prières), mais surtout du point de vue de l'intelligibilité des filiations historiques et culturelles. Parmi les Églises aladura, étudiées notamment par J. Peel ${ }^{3}$, émerge dans les années 1920, l’Église des Chérubins et Séraphins sur laquelle A. de Surgy a eu l'occasion de travailler aussi au Bénin (même si elle y est moins répandue). Or un des apports intéressants de son enquête est non seulement de rappeler qu'Oschoffa, après une éducation méthodiste, a fréquenté longuement un groupe de prières aladura et cette même Église, mais surtout que pratiquement toute la liturgie (du jardin de prière au pèlerinage de Noël sur la plage), toute l'organisation des grades et l'institution centrale du ministère de la vision, qui font aujourd'hui l'originalité des Célestes, sont reprises des Chérubins et Séraphins. Sans gommer l'importance du moment prophétique, et de la retraite initiatique où Oschoffa reçoit la révélation de sa mission, on peut considérer que l'ECC n'est au départ qu'une dissidence fomentée par un membre des Chérubins et Séraphins, exclu de cette Église pour adultère avec une femme qui deviendra la première visionnaire de la nouvelle Église, le genre d'affaire qui s'est répétée bien des fois depuis la création de l'Église.

Le refus affiché de prendre en compte l'importance de l'héritage yoruba dans la genèse de l'ECC s'éclaire d'une autre façon lorsqu'on réalise qu'A. de Surgy arrive à présenter la biographie légendaire du Prophète Oschoffa ${ }^{4}$ en en faisant un simple citoyen «béninois » (à l'époque dahoméen) et en gommant entièrement les débats sur l'origine yoruba de ses parents et le fait que ses grands-parents (Ojo et Koshina) étaient des migrants (sans doute esclaves) issus d'Abéokuta et d'Imeko, en pays Yoruba. L'artifice des frontières coloniales a séparé des populations proches et apparentées, mais ce genre d'occultation dans une histoire actuelle de l'Église signe une allégeance un peu trop marquée au point de vue de la paroisse-mère de Porto-Novo dont les leaders n'ont jamais accepté l'installation du Prophète au Nigeria et surtout le fait qu'il puisse être enterré aujourd'hui dans la Cité Céleste près de la tombe de sa mère à Imeko ${ }^{5}$. La question de l'identité du prophète n'est pas seulement « ethnique » ou ethno-nationale, même si cette dimension a pris une importance majeure avec les conflits qui entourent sa succession depuis sa mort en 1985 et la consécration d'une véritable "bicéphalie » au sein de l'Eglise. L'entrée «nigériane » que nous restituent les travaux de A. U. Adogame pourrait, à l'inverse, donner l'impression de céder aux revendications identitaires yoruba mais ce dernier tient à marquer fortement sa distance par rapport à la thèse selon laquelle la CCC est en fait « une Église yoruba ». L'important est de mieux comprendre comment l'invention prophétique originale de l'ECC s'inscrit dans toute une «matrice culturelle yoruba » et parachève l'émergence de longue date d'un christianisme aladura dont elle modifie en retour l'esprit initial. Une telle

${ }^{3}$ Peel J. D. Y., Aladura: A Religious Movement Among the Yoruba, Oxford, Oxford University Press, 1968.

${ }^{4}$ Le prophète a donné lui-même une longue version de sa biographie le 18 janvier 1969 à Makoko au Nigeria, version reprise dans le document Constitution (édité par le Diocèse du Nigeria en 1980) que A. DE SURGY ne prend pas en compte en s'en tenant à la seule version de Lumière sur le Christianisme Céleste, autre document publié et reconnu par la Paroisse-mère de Porto-Novo en 1972.

${ }^{5}$ Que A. de Surgy accorde à Benoît Agbaossi, le leader et l'héritier spirituel de la Paroisse Mère de Porto Novo, les titres de « Régent» ou de «Pasteur » auxquels il prétend, en précisant (au bout de trente pages) qu'il existe quand même d'autres prétendants à ce titre, comme le fut A. Bada, du Diocèse du Nigeria, est une chose, mais que le même Agbaossi soit régulièrement désigné comme le «Chef de l'Église » fait sérieusement problème au regard des divisions présentes. 
mise en perspective, historique et culturelle, mais aussi dialectique, est depuis longtemps engagée au Nigeria par d'autres anthropologues comme B. Ray ${ }^{6}$.

L'appellation qui semble finalement avoir la faveur d'A. de Surgy est celle d'Église " afro-chrétienne », à condition de ne pas confondre cette appellation composée avec quelque syncrétisme ou bricolage de paganisme et de christianisme. Alors que tous les écrits consacrés à cette Église (et les titres des travaux béninois cités dans la bibliographie en témoignent ${ }^{7}$ ) ne peuvent éviter de se poser la question du syncrétisme, quitte à problématiser le terme ou à le reformuler dans l'esprit de la tradition anthropologique, A. de Surgy récuse catégoriquement le terme en confortant sa définition la plus cléricale et la plus stigmatisante (mélange confus et incohérent). Sa thèse est que le Christianisme Céleste est une Église purement chrétienne simplement adaptée à la «mentalité africaine » et à ses formes d'expression coutumière, ou bien plus, une forme d'inculturation "spontanée », avant la lettre, une inculturation " par le bas », un christianisme authentique émergeant de la spiritualité africaine. Cela étant dit, cette posture théologique n'occulte pas heureusement l'interrogation anthropologique fondamentale des rapports entre les conceptions et les pratiques rituelles de la religion traditionnelle du Vodu et du $\mathrm{Fa}$ - dont $\mathrm{A}$. de Surgy est un éminent spécialiste - et le christianisme, dont le message biblique, et missionnaire, n'a rien d'univoque. Ce que l'auteur essaie de nous faire comprendre c'est que les Célestes sont à la fois de bons chrétiens, de vrais chrétiens, et que néanmoins leurs conceptions et leurs pratiques s'inscrivent dans la continuité des ressources de la tradition Vodu, un paradoxe qui ne manque pas de troubler les autres Églises qui soupçonnent quelque compromission avec le fétichisme (un christianisme avec fétiches ?), mais sur lequel l'ethnologue apporte des données et des clefs d'analyse qui méritent d'être discutées attentivement.

S'attachant à reconstituer ce que pourrait être une «théologie » céleste qui n'existe pas de façon explicite, A. de Surgy nous explique que les Célestes sont de bons chrétiens d'abord parce qu'ils croient au Diable : "Constamment célébré sous un mode négatif, par imprécations lancées contre lui, Satan se révèle, pour un Chrétien Céleste, aussi nécessaire au fonctionnement du monde que le principe de la sorcellerie pour un initié aux cultes vodous» (p. 52). Dieu intervient comme le recours suprême face aux puissances divines traditionnelles (Legba et autres) diabolisées. On est donc d'emblée dans un monde de rapports de forces, bonnes et mauvaises, qui s'inscrit dans la continuité de la vision du monde traditionnel : " C'est ainsi que la célestialité de l'ECC me paraît ne pouvoir être bien comprise qu'à la lumière des conceptions traditionnelles demeurant toujours présentes à l'arrière-plan » (p. 59). Dans ces conceptions, ce qui permet d'agir sur les évènements c'est que les énergies spirituelles, les forces saintes, qui émanent de Dieu ou de l'Esprit Saint circulent par la médiation des objets et des substances (eau bénite, bougies, huile sainte, pierres de St Michel, etc.), par l'investissement des corps qu'elles agitent (transe visionnaire), et sous le contrôle suprême du pouvoir sacré dont dispose le souverain

${ }^{6}$ RaY Benjamin C., «Aladura Christianity: A Yoruba Religion », Journal of Religion in Africa, XXIII, 3, 1993, pp. 266-291. Il faut reconnaître que l'importance des travaux anglophones consacrés ces dernières décades à la religion yoruba conduit sans doute à une surévaluation de la spécificité de son identité culturelle au regard de celle des populations apparentées.

${ }^{7}$ Citons entre autres la thèse de Sodokin CoDJo, Les syncrétismes religieux contemporains et la société béninoise - Le cas du Christianisme Céleste, doctorat de $3^{\mathrm{e}}$ cycle, Lyon, Université de Lyon II, 1984. 
de l'Église (monopole du pouvoir de l'onction). Les pratiques de voyance ou de protection qui font appel à la manipulation de ces forces saintes (visions célestes, prières de force, travaux spirituels) sont en tout point " analogues », " équivalentes », selon les termes d'A. de Surgy, aux pratiques traditionnelles.

Ce qui empêcherait de les confondre pour autant avec des pratiques que l'on dit «païennes » ou «fétichistes », c'est d'une part qu'elles ne font pas appel aux mêmes puissances que celles du Vodou, et d'autre part qu'elles sont au service exclusif de la protection des sujets, deux arguments qui ne sont pas toujours très évidents au regard même des données présentées par l'auteur. La différence substantielle entre Legba et St Michel Archange, entre les dieux vodou et les saints, est-elle à ce point significative dans un monde de forces où il n'est pas inconcevable que les puissances du Vodu se rallient à la "cause du Christ » ou que les pouvoirs divins conférés par Dieu puissent être mis au service du mal. Surtout, à défaut d'être sollicitées les puissances traditionnelles, une fois diabolisées, sont pleinement reconnues et si l'on peut dire fortifiées à tel point que sans elles «l'homme n'aurait plus besoin de se remplir d'une force divine qui s'avère indispensable à sa sanctification » (p. 52). Quant à l'usage purement protecteur des forces saintes, il est sérieusement à relativiser au regard de l'arsenal impressionnant des prières de force et de combat dont les termes patiemment transcrits par l'auteur sont d'une agressivité sans équivoque. Qu'il s'agisse de détourner la menace de mort qui pèse sur une personne sur une victime substitutive ou d'emprisonner la force maléfique dans un œuf (p. 243), la délivrance du mal passe aussi par l'élimination de l'autre persécuteur qui menace l'intégrité du sujet (et pas seulement par le pardon des péchés que demandent les prières ...).

Au-delà du débat théologique (christianisme et/ou fétichisme ?) sur lequel A. de Surgy revient avec force dans sa conclusion, la question anthropologique reste de savoir surtout ce qu'il faut conclure de ces analogies que l'auteur cultive à loisir entre conceptions ou pratiques traditionnelles du Vodou (ou du Fa) et Christianisme Céleste. Qu'il s'agisse de la Bible qui assume le même rôle que les mythes ou contes de Fa (p. 61), de la retraite initiatique du Prophète en forêt qui rappelle « étrangement » celle des chefs de culte traditionnels, ou - plus audacieux - du sacrement de l'Eucharistie présenté comme "un mystère analogue à la transformation d'un fétiche » (p. 59), l'auteur oscille entre des formulations plus ou moins savantes en termes d'équivalence fonctionnelle - pour ne pas parler d'homologie structurale et un "démon de l'analogie » qui procède, comme le dit Bourdieu, par ressemblance globale et abstraction incertaine sans toujours préciser le rapport sous lequel le rapprochement (de même que ...) est pertinent ou trouve sa limite. Le raisonnement analogique a pour vertu de ménager des rapprochements partiels et ambigus en excluant l'identification, mais comme outil d'analyse du travail symbolique, plus ou moins conscient, auquel se livrent les Célestes, il ne peut être la clef ultime. Car il faut intégrer au moins une donnée essentielle : les analogies suggérées grâce à la connaissance approfondie du Vodou et du $\mathrm{Fa}$ ont sans doute quelque écho dans l'esprit des Célestes, mais il est clair que la thèse de la continuité ou l'équivalence qu'elles sont censées étayer ne peut être admise, et est même totalement exclue, par les mêmes Célestes. Comme le dit A. de Surgy lui-même, et comme les fidèles le répètent à loisir : " l'Église du Christianisme Céleste se veut exclusivement fondée sur la Bible» (p. 59). Bien sûr, les Célestes ne se privent pas d'aller rechercher dans la vie tribale des Hébreux, ce qui justifie leur tolérance par rapport à telle pra- 
tique (la polygamie) ou le fondement de tel interdit (les couleurs rouge et noir), mais ce jeu subtil de dénégation de la reprise des traditions locales et de redécouverte du message biblique primitif obéit à une politique de double entente ou à une logique paradoxale que l'on méconnaît si l'on s'arrête à la construction savante de correspondances.

Très au fait, pour sa part, des débats les plus récents qui entourent la problématisation des processus syncrétiques, A. U. Adogame parle de politique de l'identité fondée sur la synthèse de la matrice culturelle yoruba et du message biblique. Les travaux anthropologiques qui revisitent ces dernières années la culture et la religion yoruba ne manquent pas mais en général ils s'inscrivent plutôt dans le souci de comprendre (ou d'accompagner) une renaissance ethno-nationale yoruba qui va de pair avec le mouvement " noir » américain. Des travaux comme ceux de Hallgren ${ }^{8}$ sur le concept de ase, la «force vitale », l'énergie cosmique, alimentent, malgré eux, l'idéologie de la réafricanisation des cultes ou même les spéculations de la nébuleuse du New Age. Que cette «matrice culturelle yoruba » puisse être également le creuset du christianisme Aladura confirme son extrême complexité et plasticité. Mais là encore, la «synthèse » que véhicule la CCC tire toute sa force, il faut le dire, du fait qu'elle ne se donne pas comme telle, puisqu'elle se veut l'expression pure du «Plein Évangile ». Une synthèse qui n'exclut pas la pratique d'un dualisme qui se retrouve de manière exemplaire dans la construction du charisme prophétique d'Oschoffa et dans le double titre qu'il s'est toujours donné : Prophète et Pasteur. À la différence d'autres figures prophétiques africaines comme celle d'Harris, auquel certains de ses fidèles le rattachent, Oschoffa cumule d'emblée le charisme prophétique et le statut de chef d'Église, gestionnaire des biens de salut disposant entre autres du pouvoir exclusif de l'onction qui décide de toute avancée en grade au sein de l'Église. Cette double autorité charismatique et bureaucratique est humainement assumée par un vrai père (Papa Oschoffa pour tous les Célestes) usant du pouvoir spirituel, de la force vitale (ase) et du sens du commandement (agbara) qui étaient l'apanage des chefs traditionnels et des babalawos, pour gérer la grande famille ecclésiastique (A. U. Adogame, p. 117).

Ce double jeu ou cette double entente se retrouve particulièrement au cœur du ministère de la vision auquel chacun de nos deux auteurs consacre un chapitre important. Le visionnaire céleste remplit une fonction quasi oraculaire qui n'a rien à voir, comme tient à le souligner A. de Surgy, avec la fonction expressive de la glossolalie (le plus faible des dons pour tous les Aladura). A. U. Adogame note pour sa part que le prophétisme itinérant du messager de Dieu, porte-parole des révélations divines, très présent dans les premières Églises Aladura, a laissé place dans la CCC à une fonction de voyance et de consultation divinatoire. À la différence néanmoins de la géomancie du Fa qui passe par l'interprétation des signes et des figures, mais à l'image de la transe maîtrisée des vodushi, les secousses du corps et la voix syncopée sont ici le seul véhicule de la parole de l'Esprit. La transcription des visions, dont A. de Surgy nous présente tout un corpus, introduit en fait rapidement à une liste de prescriptions ou de «travaux spirituels » (exposition du corps, bains et prières de combat) qui évoquent ceux que l'on accomplit dans les « couvents du Vodu », la différence se jouant entre autres sur la couleur des bou-

${ }^{8}$ Hallgreen R, The Vital Force: A Study of Ase in the Traditional and Neo-traditional Culture of the Yoruba People, Lund, Lund Studies in African and Asian Religions, vol. 10, 1995. 
gies utilisées ou la manière de casser les œufs ... La vision est enfin une grâce de l'Esprit qui est officiellement gratuite mais l'économie de la voyance ouvre sur un marché de biens de salut et de guérison (bougies, encens, fruits, eau sacrée, parfum de St-Michel, etc.) qui peut transformer le visionnaire en marchand de fétiches. Seule la prière, la sanctification régulière et la fréquentation quotidienne des méandres de la Bible sont susceptibles de renforcer et de maîtriser le don de vision, de fournir avec le temps l'intelligence de la parole divine et d'éviter les pièges et les tentations du fétichisme.

Expression suprême de la manifestation de la puissance de l'Esprit Saint, la vision est en même temps l'objet par excellence du soupçon, le lieu de la duplicité ou de la confusion entre le Diable et le bon Dieu. Les mises en garde réitérées du Prophète fondateur que rappelle A. U. Adogame dans son souci d'intégrer les tensions historiques et les contradictions internes de l'Église, montrent que l'ECC a en fait toujours été travaillée par l'obsession d'épurer la vision de ses attaches païennes en clarifiant les rapports entre prophétie et voyance, vision et possession, sanctification et fétichisme. Cette lutte symbolique, comme nous l'avons observé, traverse les visionnaires eux-mêmes ou opposent ces derniers aux gestionnaires de l'institution. Dans les terres de mission que sont le Gabon et le Congo, à New York ou à Londres, comme au cœur du Nigeria ou du Bénin, des «virtuoses » de la vision, souvent de jeunes lettrés migrants, des intellectuels chômeurs, à l'image des initiateurs du mouvement Aladura des années trente, interpellent les "mamans » qui ont fait de la consultation visionnaire leur affaire ou pourchassent les fauxprophètes, les babalawo nigérians ou les bokönö béninois qui exercent à leur compte ${ }^{9}$.

La manière dont cohabitent l'attachement aux ressources d'une culture divinatoire traditionnelle et la conversion aux vertus du prophétisme des Églises de l'Esprit reste troublante et objectivement ambiguë. Tout dans cette pratique visionnaire fait penser, on l'a dit, au système divinatoire du culte de Fa qui était le noyau de la religion yoruba, et en même temps il n'en reste rien. Aucun dispositif divinatoire de tablettes et de noix palmistes, ou autres, aucune combinatoire des signes à déchiffrer, aucune connaissance ésotérique, n'interviennent ici. L'habitus initiatique et la maîtrise savante de la combinatoire des 256 couples de figures géomantiques ont laissé la place à la connaissance experte du texte biblique et aux jeux de miroir des citations de versets. Les noms des orisha sont remplacés par l'invocation répétitive des noms saints de Jehovah ou de Jésus-Christ, ou par ceux des anges, saint Michel, saint Gabriel, saint Raphaël ou saint Uriel. Les sacrifices aux dieux et les travaux des couvents du Vodu sont relayés par les offrandes de fruits, la flamme des bougies et la fumée de l'encens, l'imposition de l'huile sainte et la purification de l'eau bénite, mais surtout par l'efficacité absolue de la prière.

\footnotetext{
${ }^{9}$ MARY André, «Culture pentecôtiste et charisme visionnaire au sein d'une Église indépendante africaine ", Archives de Sciences Sociales des Religions, n 105, 1999, pp. 29-50. Alfred DE SuRGY souligne qu'au Bénin le ministère de la vision est en grande majorité une affaire de femmes, les statuts de leaders ou d'évangélistes étant réservés aux hommes, mais les "grands visionnaires » qui assurent la direction spirituelle sont également tous des hommes, une division du travail «calquée » selon lui sur le Vodou (op. cit., p. 208).
} 
En l'absence d'une continuité matérielle, toujours masquée et déniée, la simple référence à une similitude de fonction fondée sur l'attachement à une religiosité pragmatique qui répond aux problèmes de la vie quotidienne (santé, travail, fécondité, comme en témoignent tous les récits de conversion rapportés par A. de Surgy), suffit-elle à étayer la thèse de la continuité par rapport aux attitudes traditionnelles ? Comme le dit C. Lévi-Strauss, de telles explications fonctionnalistes confinent au truisme. On peut faire remarquer que la culture pentecôtiste fonctionne elle-même traditionnellement sur la " résolution de problèmes », et que c'est surtout un point fort du néo-pentecôtisme actuel. Il faudrait par contre faire toute sa place à l'ambivalence qui travaille ces pratiques visionnaires et interroger de plus près ce que devient, au cœur de la consultation et du diagnostic individuel des maux, la conception de la personne et du destin individuel, la configuration des forces invisibles, bénéfiques et maléfiques, qui étaient au fondement du dispositif divinatoire du $\mathrm{Fa}$.

L'ECC comme la plupart des Églises indépendantes africaines accordent une grande place, en dehors des cultes ordinaires qui sont consacrés à la délivrance des maux, et particulièrement aux problèmes de fécondité, à l'ensemble des rites de passage qui accompagnent la vie d'une personne, de la naissance à la mort : rite de sortie de l'enfant ou levée de deuil. Ce sont des lieux ou des moments où se négocient de façon très serrée toutes sortes d'arrangements entre les conceptions traditionnelles du corps et de la personne, les prescriptions bibliques et l'ordre rituel chrétien. Ainsi les Célestes sont connus (et mal vus à ce sujet) pour refuser d'admettre la présence impure des cadavres dans l'enceinte sacrée de l'église et de la paroisse, les rites d'enterrement conduisant directement du domicile du défunt (ou de la morgue) à la tombe du cimetière. Mais lors des cultes commémoratifs et des levées de deuil qui suivent (aux $8^{\mathrm{e}}$ et $40^{\mathrm{e}}$ jours), l'usage substitutif d'un vieux matériel chrétien, le catafalque, assume, à la place des autels des ancêtres (assin) ou des masques (egungun) traditionnels, une sorte de présence et d'accueil symbolique du mort (de son esprit ou de son âme) dans le lieu saint, le « sein d'Abraham " ${ }^{10}$. Le même genre de pratique en double se retrouve à la naissance, lors de la sortie de l'enfant au septième jour, puisque cette fois c'est la mère encore impure qui ne peut entrer dans l'église. Une "devancière » ou une « visionnaire » porte l'enfant à sa place.

Dans la description des rituels qui occupe une place centrale et stratégique dans les monographies d'Église africaine et particulièrement dans les deux ouvrages en question, l'ethnographe ne peut se contenter, comme on le faisait dans un contexte de tradition orale où le texte c'est le rite, de transcrire les séquences rituelles observées ou relatées sans donner l'impression de redoubler le travail d'écriture des clercs. Les rares écrits qui circulent dans les Églises africaines sont en effet principalement consacrés à l'organisation du culte et à la Constitution, de même qu'aux prescriptions et ordonnancements de la liturgie, une symbiose remarquable $\mathrm{du}$ ritualisme de la tradition africaine et du légalisme bureaucratique moderne. L'enjeu anthropologique de la description des rites se situe plus que jamais sur le terrain de l'interprétation ou de ce que C. Geertz nomme la «description dense »

${ }^{10}$ Signalons sur ces sujets l'excellent mémoire universitaire de J. Noret : L'Église Invisible. Deuil, souci, et statut des morts chez les Chrétiens Célestes du Sud-Bénin, Bruxelles, Université Libre de Bruxelles, 2001. 
(thick description), la restitution des couches sédimentées de signification qui travaillent les objets et les gestes mobilisés.

La présentation d'un même rituel comme celui de la sortie de l'enfant par A. de Surgy et A. U. Adogame est de ce point de vue pleine d'enseignements. Le premier parle de «la présentation d'un bébé au temple » où la référence biblique à la circoncision des juifs, dans un contexte où il n'est pas question de circoncision proprement dite, "sert de substitut au rite traditionnel de présentation d'un nouveau né, une semaine après sa naissance, dans la cour de l'habitation familiale » (p. 123). Par rapport à ce que décrit ou prescrit la brochure Ordre des cultes et des cérémonies éditée par le Siège de Porto-Novo, la lecture insiste particulièrement sur la place symbolique du «plateau à sept bougies » (qui a pour pendant la corbeille à sept fruits) qui accompagne l'entrée du cortège et la porteuse de l'enfant dans l'église, trois bougies étant au départ allumées, puis les quatre autres seulement une fois au cœur du temple. La symbolique du chiffre sept $(3+4)$ est omniprésente comme partout dans la liturgie céleste et les spéculations ésotériques de l'ECC. Mais c'est surtout la gestion des substances et des matières (l'eau, le sel, le miel, présentés trois fois sur les lèvres de l'enfant) qui forme le rite spécifique du moment. Du plateau des sept bougies, six seront récupérées pour le culte réservé aux femmes stériles du mercredi matin et l'eau qui a servi à éteindre les bougies sera mélangée à l'eau de toilette de l'enfant. Le «bougisme » est ici le substitut du fétichisme et organise la circulation de la substance sacrée.

Le même rite est désigné par A. U. Adogame comme "the rite of naming » (Esin Isomoloruko, en yoruba), ce qui met l'accent au cœur du rite spécifique du versement de l'eau sur les lèvres sur l'enjeu de la nomination de l'enfant (p. 165). Le nom, ou plus exactement le prénom, est censé être révélé auparavant par un visionnaire, et c'est d'ailleurs également un visionnaire (ou une visionnaire selon le sexe de l'enfant) qui porte dans ses bras l'enfant dans le temple. Le commentaire n'oublie pas l'évocation biblique de la présentation de Jésus au temple, mais il souligne surtout l'importance dans le monde yoruba de la consultation d'Ifa par le babalawo et de la divination du nom de l'enfant qui l'associe à tel ou tel orisa, et décide en définitive de son identité.

Le travail de synthèse auquel se réfère A. U. Adogame - travail qu'il illustre également dans son analyse de l'Eucharistie céleste (p. 212) - relève surtout d'une double réinterprétation croisée, dialectique, du christianisme par la tradition yoruba et des rites yoruba par des significations chrétiennes. Cette possibilité constante d'une double lecture de la liturgie, en deçà des justifications bibliques mises en avant, dépend, on le voit, du contexte culturel privilégié et nul doute que les résonances individuelles que comportent les objets, gestes ou séquences rituelles pour les sujets concernés sont en fait d'une grande variation. En l'absence de toute théologie explicite, les guides liturgiques s'en tenant aux prescriptions minimales (prières, psaumes, prédication, chants), les possibilités de surinterprétation théologique nourries de la connaissance anthropologique des cultures locales sont également évidentes.

L'inscription des Églises prophétiques africaines dans les sociétés locales et nationales qui les ont vu naître et les opportunités d'internationalisation qui s'offrent à elles, en grande partie par le biais de la migration, constituent un autre enjeu majeur des deux études en présence. 
Le Prophète Oschoffa avait déclaré : «L'Église du Christianisme Céleste n'est la propriété d'aucune race, d'aucun pays et de personne. C'est l'Église de JésusChrist, une Église Universelle ». Mais aux yeux de « la petite nation béninoise », pour reprendre les termes d'A. de Surgy, le discours céleste, confronté au péril de "l'éléphant yoruba », cède souvent à la logique de "l'autochtonie ». Le chef du Diocèse du Bénin a vocation à être le chef naturel de l'Église mondiale. Les hommes aux soutanes blanches et aux pieds nus, le pèlerinage de Sémé, sont des éléments du paysage et du patrimoine national. Chaque quartier, chaque village (au moins sur la côte, Cotonou et la région de Porto-Novo) a son église. Mais cette dimension quasi-officielle et cette banalisation de l'image des Célestes ne confèrent pas pour autant à cette Église la légitimité dont disposent les Églises catholique, protestante ou méthodiste. Il faut dire que la situation effective de "schisme» entre le Bénin et le Nigeria, avec destitution et excommunication réciproque des responsables, les scandales liés aux pratiques douteuses des vrais-faux visionnaires, aux prétendus miracles ou aux sacrifices, les affaires de corruption dans la gestion des paroisses que la presse locale véhicule et dont elle se gausse, n'arrangent pas l'image des «soutanés ». Manifestement les gouvernements successifs (Kérékou I, Soglo ou Kérékou II) se sont lassés d'intervenir pour faciliter les réconciliations entre "factions ", à force d'être accusés d'ingérence ou de trahison par les uns et les autres.

Comme le reconnaît A. de Surgy, la «grande famille » de l’Église Céleste du Bénin n'a rien de monolithique, elle n'a cessé d'être soumise à la segmentation. Les dissidents, les exclus, les non-orthodoxes, les oscillants, les repentis de tel ou tel camp sont nombreux. L'enjeu se situe rarement sur le plan théologique ou liturgique mais se nourrit de conflits de pouvoir et de querelles de chefs paroissiaux constamment traduits en termes de sorcellerie ou de conspiration diabolique ${ }^{11}$. Même entre Porto-Novo, la paroisse mère, une sorte de " petit Vatican », et Cotonou, la grande ville aux nombreuses et grandes églises, les tiraillements sont nombreux, la compétition vive. Toute l'histoire nationale a été dominée, notamment après la mort du Prophète, par les déchirements du fameux triumvirat béninois, les trois « responsables nationaux », au départ ligué contre l'autre nigérian et intriguant Bada : Agbaossi, «l'ancien »; Gonçalvès, le « secrétaire » ou « le professeur », et Tiamou, l'aide de camp de Kérékou I, qui porte tout le poids de la «trahison » et des « revirements » qui ont fait éclater l'entente. Le conflit de tendance entre Bada et Agbaossi a déchiré l'Église béninoise elle-même.

La majorité des béninois reste néanmoins fidèle à la tendance portonovienne et agbaossiste. Il faut dire qu'il est devenu difficile, surtout à partir des années 1996-1997 de se dire ou de s'afficher pro-Bada au Bénin, sans passer pour un traître non seulement à son Église et à son prophète mais tout simplement à son pays, terre sainte de cette Église. Compte tenu des enjeux financiers que comporte le choix des lieux de pèlerinage et de la «bénédiction divine » que représente l'afflux de devises lié au marché de l'onction, ceux qui détournent les Célestes du monde entier vers Imeko au Nigeria sont accusés de commettre un « crime économique contre la nation béninoise ».

${ }^{11}$ Sur ces questions de dissidence, Cf. HenRy C., « Du vin nouveau dans de vieilles outres : parcours d'un dissident du Christianisme Céleste », Social Compass, vol. 48 (3), 2001, pp. 353-368. 
Le Diocèse du Nigeria, qui a ses propres dissidences et ses propres procès de succession exploités par les médias, ne doute pas de sa prétention à diriger une Église universelle qui se veut plus ouverte sur le monde et la modernité. L'Église nigériane n'exclut pas cependant le compromis puisqu'elle n'a jamais nié que le Siège Mondial devait se maintenir à la Paroisse Mère de Porto-Novo. Les notions de « Paroisse Mère », de «Saint-Siège », aux résonances très romaines, continuent à jouer un rôle symbolique important dans le discours de l'unification. La thèse d'A. U. Adogame est que Bada, le successeur nigérian du Prophète, a poursuivi en tant que Suprême Évangéliste, le processus de « routinisation du charisme » déjà engagé du vivant du fondateur. Sous l'influence de cet homme d'institution appelé à gérer le dépôt sacré, la CCC a renforcé son organisation bureaucratique à l'échelle mondiale (paroisse, district, diocèse, comité mondial). Le pasteur Bada a eu une politique systématique de voyage et de visite dans les paroisses du monde entier (Londres, Paris) et d'exploitation des medias par l'enregistrement télévisé des cultes, la diffusion de cassettes des sermons, l'ouverture de sites Internet (à Londres et à New York). Le caractère missionnaire de l'Église est désormais explicitement affirmé par la nomination d'émissaires pastoraux chargés de gagner de nouveaux pays (même la Chine...), de même que par l'organisation volontariste de campagnes d'évangélisation et de réveil. Le souci de la formation biblique des évangélistes donne lieu à l'ouverture d'écoles, de séminaires et même d'un institut de théologie au Nigeria. En un mot, sur le terrain anglophone, comme le souligne A. U. Adogame (p. 84), l'Église s'affiche nettement comme une Église charismatique et évangélique vivant à l'heure du village global et cherchant à mettre sous le boisseau la dimension ritualiste et fétichiste liée à son ancrage paroissial et africain.

En réalité, dans chacun des camps en présence, pro-béninois et pro-nigérian, les vieux leaders ont dû faire face à la contestation des jeunes lettrés, souvent visionnaires, qui se sont organisés en mouvement de la «Jeunesse Chrétienne Céleste ». Les thèmes de leur programme tournent autour de la «moralisation de la vie de l'Église », des conditions spirituelles de l'exercice de la vision et de l'importance de la formation biblique, et bien sûr de la dénonciation du commerce de l'onction (de sa suppression éventuelle) et de la gestion corrompue des comités paroissiaux (lieux de concentration des notables). L'exigence de purification et de rationalisation dont ces jeunes sont porteurs ne les conduit pas toujours à des positions œcuménistes; elle trouve dans le discours de la régénération " ethno-nationale » et l'obsession de la contamination par l'autre (béninois ou nigérian, selon les camps) les voies de son affirmation ${ }^{12}$.

En définitive, le conflit de succession, la compétition qu'il encourage en matière d'implantation paroissiale et même l'affrontement des identités nationales qu'il a réveillé, ont beaucoup contribué dans la dernière décade à l'expansion et à la transnationalisation de l'Église. Comme pour d'autres Églises africaines (harriste, kimbanguiste) la logique de la reconnaissance tend à imposer la conformité

${ }^{12}$ Le journal béninois La Dernière Barque, qu'A. de Surgy utilise abondamment comme une source autorisée, émane en fait d'un groupe de la «Jeunesse Chrétienne Céleste » qui s'est d'abord affiché comme indépendant et s'est fait connaître par des positions critiques et des propos réformistes adressés initialement à toute l'Église, mais qui a fini par basculer manifestement, surtout à partir de la rupture de 1996, dans le camp porto-novien et par soutenir des positions ethno-nationalistes parfois virulentes. 
aux normes d'une religion mondiale en renforçant le contrôle bureaucratique, la codification des croyances et des pratiques.

A. de Surgy considère que l'ECC représente une forme d'inculturation spontanée du christianisme qui pourrait servir de leçon aux théologiens en quête d'adaptation du message évangélique à la mentalité africaine. La synthèse éclairée ou l'expertise théologique que propose en conclusion l'anthropologue en cherchant à convaincre les uns et les autres (à commencer par les Célestes) que le Vodu et le Christianisme sont d'une certaine façon équivalents, n'engage évidemment que son auteur. Mais puisque nous sommes aussi invités à nous prononcer par rapport aux valeurs d'un christianisme africain qui viendrait suppléer aux défaillances et aux impasses de l'humanisme occidental par ses vertus de tolérance, on peut quand même se demander en quoi cette religion des forces saintes (avec ses bougies, ses onctions d'huile et ses odeurs d'encens, et son «parfum de Saint Michel»...) entièrement mobilisée par son combat contre Satan et les sorciers qui se cachent parmi nous, apporte des « leçons d'humanisation de la vie sociale» (p. 304) ? Bien plus, peut-on vraiment faire passer la "communauté céleste », cette société paroissiale totalement hiérarchisée, dominée par le pouvoir sacré d'un souverain, excluant les femmes (trop impures) de tout accès aux fonctions supérieures, et traitant toute dissidence comme une manifestation du démon, comme une formule providentielle répondant aux «carences de notre organisation sociale » (p. 303)?

Tout le monde peut faire le constat que dans l'Afrique d'aujourd'hui, la fin des régimes de parti unique, le retrait ou la faillite de l'État dans les domaines de l'éducation, de la santé et de la sécurité des populations, le désengagement par rapport aux associations syndicales ou politiques, favorisent le développement et la multiplication des Églises qui s'offrent comme les seuls refuges communautaires. Mais de là à considérer cette situation comme pleine de promesses et à prôner le modèle d'Église comme relais de la cité-État, il y a un grand pas. Même sur le terrain des « politiques de l'identité », où les États-nations n'offrent plus de ressources d'identification crédibles face au défi de l'ethnisme, les prétentions des Églises africaines à être porteuses d'un message universel ouvert à tous les «frères en Christ » ne réussissent pas toujours à passer les frontières. Toute l'histoire de l'entreprise de mondialisation d'une Église comme l'ECC versus CCC montre malheureusement de façon exemplaire que celle-ci n'a pas réussi, à ce jour, à surmonter ses démons ethno-nationaux ${ }^{13}$.

\author{
André MARY \\ Institut d'Études Africaines - MMSH \\ Aix-en-Provence
}

${ }^{13}$ Nous nous permettons sur ce sujet de renvoyer à notre article, MARY André, «Anges de Dieu et esprits territoriaux : une religion africaine à l'épreuve de la transnationalisation », Autrepart (14), 2000, pp. 71-89. 